\title{
Formation and dynamics of self-sustained neutron haloes in disk accreting sources
}

\author{
A. A. Belyanin ${ }^{1,2}$ and E. V. Derishev ${ }^{1,3}$ \\ 1 Institute of Applied Physics, Russian Academy of Science, 46 Ulyanov st., 603950 Nizhny Novgorod, Russia \\ 2 Dept. of Physics, Texas A\&M University, College Station, TX 77843-4242, USA \\ e-mail: belyanin@atlantic.tamu.edu \\ 3 MPI für Kernphysik, Saupfercheckweg 1, 69117 Heidelberg, Germany
}

Received 21 August 2001 / Accepted 1 October 2001

\begin{abstract}
It has been recognized long ago that the presence of hot plasma in the inner accretion disks around black holes could lead to the neutron production via dissociation of helium nuclei. We show that, for a broad range of accretion parameters, neutrons effectively decouple from protons and pile up in the inner disk leading to the formation of self-sustained halo. This means that new neutrons in the halo are supplied mainly by the splitting of helium nuclei in their collisions with existing neutrons. Once formed, such a halo can exist even if the proton temperature is much lower than the energy threshold of helium dissociation. We show that neutron haloes can be the natural source of relativistic electrons and positrons, providing characteristic comptonization spectra and hard spectral tails observed in many black hole candidates, and also giving rise to relativistic outflows. Deuterium gamma-ray line at $2.2 \mathrm{MeV}$ resulting from neutron capture is also expected at a level detectable by future INTEGRAL mission. Furthermore, the presence of a neutron halo strongly affects the dynamics of accretion and leads to the rich variety of transient dynamical regimes.
\end{abstract}

Key words. accretion, accretion disks - nuclear reactions, nucleosynthesis, abundances - X-rays: binaries

\section{Introduction}

There are many theoretical and observational indications that the hot plasma of ion temperature $T_{\mathrm{i}}$ exceeding several $\mathrm{MeV}$ can exist in the inner accretion disks around black holes and, possibly, neutron stars. Formation of a two-temperature region with $T_{\mathrm{i}} \gg T_{\mathrm{e}}$ in accretion disks has been predicted as long ago as in 1976 (Shapiro et al. 1976). When the energy transfer from ions to electrons is slow as compared with the accretion timescale, an accretion flow becomes nearly adiabatic, and the ion temperature can reach virial values. This idea has led to the prediction of advection-dominated accretion flows which seems to find support in observations of several X-ray transients (Narayan et al. 1996, 1997) and other sources showing low radiation efficiency and high temperature of the flow.

Strong observational support for the existence of hightemperature plasma comes from gamma-ray and radio astronomy. Very hard spectra extending to $\mathrm{MeV}$ region were detected from several Galactic X-ray sources presumably containing black holes. Transient line-like features around $0.5 \mathrm{MeV}$ indicate an efficient mechanism of positron

Send offprint requests to: E. V. Derishev, e-mail: derishev@mpi-hd.mpg.de production (Bouchet et al. 1991; Churazov et al. 1993). Non-thermal radio emission and episodic ejection of relativistic jets observed in microquasars (Mirabel \& Rodríguez 1998) are definite signatures of ultrarelativistic electrons and/or electron-positron $\left(\mathrm{e}^{-} \mathrm{e}^{+}\right)$plasma and were interpreted several times as radiation-pressure dominated $\mathrm{e}^{-} \mathrm{e}^{+}$outflows (Li \& Liang 1996; Belyanin 1999).

The presence of hot ions in accretion disks leads to the rich variety of inelastic nuclear interactions. The most prominent of them is neutron production which primarily occurs through dissociation of helium nuclei. This process has potentially important observational implications arising e.g. from possibility of radiative neutron capture with subsequent formation of gamma-ray lines (Aharonyan \& Sunyaev 1984).

The main source of free neutrons in accretion disks is dissociation of helium nuclei due to inelastic collisions with energetic ions contained in the disk or free neutrons passing through it. Neutron production in accretion disks was considered several times (Aharonyan \& Sunyaev 1984; Guessoum \& Kazanas 1989) under the assumption that neutrons are quickly thermalized in the disk, advected by the bulk plasma motion and transported into a compact object on the disk viscous timescale. In this case the 
neutron fraction in the accretion flow is inevitably small and the radiative-capture gamma-ray lines are weak and smeared out by a hot rapidly rotating disk.

However, if plasma in the accretion disk is sufficiently rarefied, then the neutron and ion components in the disk effectively decouple, allowing for the accumulation of neutrons. This can lead to the formation of neutron haloes with much larger neutron number than in previously considered cases. It is important that, once formed, such a halo becomes self-sustained. This means that, even if the ion temperature in the disk falls below the threshold for He dissociation by protons or the proton density becomes too small for proton-induced dissociation to be efficient, the neutron production is supported by collisions of energetic neutrons from the halo with helium nuclei.

In this paper we propose and explore the scenario of neutron halo formation and show that it can lead to much more pronounced observational and dynamical effects as compared with previous predictions.

\section{Neutron pile-up and formation of halo}

Let us consider bulk radial motion of neutrons which is the result of angular momentum losses caused by elastic collisions with ions (mostly protons) in the accretion disk. Given the Keplerian relation of the orbital radius to the bulk orbital velocity, $R=\frac{R_{\mathrm{g}}}{2} \frac{c^{2}}{V_{\mathrm{n}}^{2}}$, one directly obtains the following expression:

$$
V_{\mathrm{n}}^{(r)}=-\frac{\mathrm{d}}{\mathrm{d} t}\left(\frac{R_{\mathrm{g}}}{2} \frac{c^{2}}{V_{\mathrm{n}}^{2}}\right)=-\frac{R_{\mathrm{g}}}{2} \frac{c^{2}}{V_{\mathrm{n}}^{3}} \nu_{\mathrm{pn}}\left(V_{\mathrm{n}}-V_{\mathrm{d}}\right) .
$$

Here $V_{\mathrm{n}}$ and $V_{\mathrm{n}}^{(r)}$ are the orbital and radial velocities of neutrons (it was assumed that $V_{\mathrm{n}}^{(r)} \ll V_{\mathrm{n}}$ ), $R_{\mathrm{g}}$ the Schwarzschild radius of the compact object, and $c$ the speed of light. The time derivative of $V_{\mathrm{n}}$ in the above expression was substituted by $-\nu_{\mathrm{pn}}\left(V_{\mathrm{n}}-V_{\mathrm{d}}\right) / 2$, where $\nu_{\mathrm{pn}}$ is the proton-neutron collision rate and $V_{\mathrm{d}}$ the disk orbital velocity.

As the orbital velocity of neutrons is larger than that of the accretion disk, the excessive centrifugal force has to be balanced by the friction force, which originates from the difference of radial velocities of the neutrons and the disk. This gives another relation:

$$
\frac{V_{\mathrm{n}}^{2}-V_{\mathrm{d}}^{2}}{R}=\frac{\nu_{\mathrm{pn}}}{2}\left(V_{\mathrm{n}}^{(r)}-V_{\mathrm{d}}^{(r)}\right) \text {. }
$$

Here $V_{\mathrm{d}}^{(r)}$ is the radial velocity of the disk and $R$ the current orbital radius. Assuming $V_{\mathrm{n}} \simeq V_{\mathrm{d}} \simeq V_{0}$ ( $V_{0}$ is the Keplerian velocity), one finds the expression for the radial velocity of neutrons:

$$
V_{\mathrm{n}}^{(r)}=-\frac{1}{4}\left(\frac{R}{V_{0}} \nu_{\mathrm{pn}}\right)^{2}\left(V_{\mathrm{n}}^{(r)}-V_{\mathrm{d}}^{(r)}\right) .
$$

The value $\left(R / V_{0}\right) \nu_{\mathrm{pn}}$ in the above equation is the number of collisions a neutron undergoes as it completes one orbital revolution, divided by $2 \pi$.
The collision rate $\nu_{\mathrm{pn}}$ may be expressed in terms of the disk optical depth for proton-neutron collisions, $\tau_{\mathrm{d}}$, which is equal to the average number of collisions experienced by a neutron passing through accretion disk with Keplerian velocity directed perpendicular to the disk plane. Given the heights of ion and neutron "disks", $h_{\mathrm{d}}$ and $h_{\mathrm{n}}$ respectively, and assuming that the cross-section of proton-neutron collisions is inversely proportional to their relative velocity, one has $\nu_{\mathrm{pn}}=\langle\sigma V\rangle_{\mathrm{pn}} n_{\mathrm{p}}\left(h_{\mathrm{d}} / h_{\mathrm{n}}\right)=$ $\left(V_{0} / h_{\mathrm{n}}\right)\left(\langle\sigma V\rangle_{\mathrm{pn}} / V_{0}\right) n_{\mathrm{p}} h_{\mathrm{d}}=\left(V_{0} / 2 h_{\mathrm{n}}\right) \tau_{\mathrm{d}}$. Finally, the expression for the radial velocity of neutrons takes the following form:

$$
V_{\mathrm{n}}^{(r)}=\frac{\left(R / 2 h_{\mathrm{n}}\right)^{2} \tau_{\mathrm{d}}^{2}}{4+\left(R / 2 h_{\mathrm{n}}\right)^{2} \tau_{\mathrm{d}}^{2}} V_{\mathrm{d}}^{(r)} .
$$

Neutrons start to pile up in the inner parts of accretion disk when $V_{\mathrm{n}}^{(r)}$ is considerably smaller than $V_{\mathrm{d}}^{(r)}$, i.e., when $\tau_{\mathrm{d}} \lesssim 4 h_{\mathrm{n}} / R$. In the limiting case of neutron halo $\left(2 h_{\mathrm{n}} \sim R\right)$ the last condition gives $\tau_{\mathrm{d}} \lesssim 1$.

\section{Criteria of neutron halo existence}

The neutrons in accretion disk pile up only when the accretion rate $\dot{M}$ is not too large, $\dot{M} \lesssim \dot{M}_{\max }$, where

$$
\begin{aligned}
\dot{M}_{\max } & =\frac{8 \pi V_{\mathrm{d}}^{(r)} V_{0} h_{\mathrm{n}}}{\langle\sigma V\rangle_{\mathrm{pn}}} m_{\mathrm{p}} \\
& =\frac{4 \pi \alpha R_{\mathrm{g}} c^{2}}{\langle\sigma V\rangle_{\mathrm{pn}}}\left(\frac{h_{\mathrm{d}}}{R}\right)^{2}\left(\frac{h_{\mathrm{n}}}{R}\right) m_{\mathrm{p}}
\end{aligned}
$$

Here we used the usual assumption $V_{\mathrm{d}}^{(r)}=\alpha\left(h_{\mathrm{d}} / R\right)^{2} V_{0}$, and the continuity equation to find the proton number density. The ratio $h_{\mathrm{d}} / R$ is treated as a parameter. The limiting accretion rate $\dot{M}_{\text {max }}$ approximately equals to that corresponding to the Eddington luminosity, provided $\alpha=0.1$ and $h_{\mathrm{d}} \simeq R$.

In the pile-up regime, the neutron-to-proton ratio in the disk, which is proportional to $V_{\mathrm{d}}^{(r)} / V_{\mathrm{n}}^{(r)} \propto \tau_{\mathrm{d}}^{-2}$, grows with decreasing accretion rate as $\dot{M}^{-2}$ and soon exceeds unity $^{1}$, so that collisions with neutrons become the main cause of helium dissociation. Actually, it happens at a lower neutron-to-proton ratio, because the neutron component tends to have higher temperature than the ion one. Even in the absence of additional mechanisms that act specifically to heat neutrons (e.g., precession of neutron's orbit in the gravimagnetic field of a rotating compact object), their temperature exceeds the ion temperature by a factor $7 / 5$.

In view of the above-mentioned, the most interesting is the self-sustained mode of neutron halo formation, when new neutrons in the halo are supplied predominantly by

1 The dependence $n_{\mathrm{n}} / n_{\mathrm{p}} \propto \dot{M}^{-2}$ may not hold when $n_{\mathrm{n}} / n_{\mathrm{p}} \gg 1$, since in this case there are processes which can lead to neutron escape on a shorter-than-hydrodynamic timescale, e.g. neutron evaporation or random-walk diffusion resulting from finite change of orbital parameters in each collision. 
the splitting of helium nuclei in their collisions with existing neutrons. This mode implies that at least one of three neutrons breaks helium nucleus before decaying or being advected into the black hole. These two conditions provide, respectively, lower and upper bounds on the range of accretion rates in which the self-sustained neutron halo may exist.

Let us consider the upper boundary first. To do this, we calculate the average number of collisions with protons experienced by each neutron on its way into the black hole:

$N_{\mathrm{c}}=\frac{\nu_{\mathrm{pn}} R}{V_{\mathrm{n}}^{(r)}}=\frac{2 \pi R_{\mathrm{g}} c^{2}}{\langle\sigma V\rangle_{\mathrm{pn}} \dot{M}}\left(4+\left[\frac{R}{V_{0}} \nu_{\mathrm{pn}}\right]^{2}\right) \frac{h_{\mathrm{n}}}{R} m_{\mathrm{p}}$,

assuming zero helium mass fraction $\eta_{\mathrm{He}}$. In the following considerations we neglect the term $\left(R / V_{0}\right) \nu_{\mathrm{pn}}$ as it is important only if $\dot{M} \simeq \dot{M}_{\max }$. The self-sustained neutron halo requires

$N_{\mathrm{c}}>\frac{1}{3} \frac{4}{\eta_{\mathrm{He}}} \frac{\sigma_{\mathrm{pn}}}{\sigma_{\mathrm{d}}} \Rightarrow \dot{M}<\frac{3 \eta_{\mathrm{He}}}{2 \alpha} \frac{\sigma_{\mathrm{d}}}{\sigma_{\mathrm{pn}}}\left(\frac{R}{h_{\mathrm{d}}}\right)^{2} \dot{M}_{\max }$

where $\sigma_{\mathrm{pn}}$ the proton-neutron scattering cross-section, having the value $\sigma_{\mathrm{pn}} \simeq 700 \mathrm{mb}$ at the energies typical for the edge of neutron halo, and $\sigma_{\mathrm{d}} \simeq 100 \mathrm{mb}$ is the dissociation cross-section in $n-\alpha$ collisions which, in effect, is the sum of cross-sections for all possible spallation processes. It is clear that the self-sustained mode maintains up to $\dot{M} \simeq 0.4 \dot{M}_{\max }$ or even higher; at still higher accretion rates inelastic $p-\alpha$ collisions are frequent enough to ensure complete dissociation of helium.

At very low values of $\dot{M}$ the neutrons may be advected so slowly that their finite lifetime, $t_{\mathrm{n}} \simeq 900 \mathrm{~s}$, comes into play. The decay losses are inevitable (and irreversible), but they become a limiting factor only if there are no other processes to compete with, for example, evaporation of neutrons from the disk. This means that the ion temperature should be considerably lower than the virial value or, in other words, $h_{\mathrm{d}} \ll R$. Keeping in mind this restriction, one may derive the absolute lower limit on the accretion rate (the one that still permits the self-sustained neutron halo) from the condition

$\frac{1}{4} \eta_{\mathrm{He}} \frac{\sigma_{\mathrm{d}}}{\sigma_{\mathrm{pn}}} \nu_{\mathrm{pn}} t_{\mathrm{n}}>\frac{1}{3}$

which gives

$$
\begin{aligned}
\dot{M}>\dot{M}_{\min } & =\frac{16 \pi}{3} \frac{\sigma_{\mathrm{pn}}}{\sigma_{\mathrm{d}}} \frac{R h_{\mathrm{n}} V_{\mathrm{d}}^{(r)}}{\eta_{\mathrm{He}}\langle\sigma V\rangle_{\mathrm{pn}} t_{\mathrm{n}}} m_{\mathrm{p}} \\
& =\frac{4}{3 \sqrt{2}} \frac{\sigma_{\mathrm{pn}}}{\eta_{\mathrm{He}} \sigma_{\mathrm{d}}} \frac{R_{\mathrm{g}}}{c t_{\mathrm{n}}}\left(\frac{R}{R_{\mathrm{g}}}\right)^{3 / 2} \dot{M}_{\max } .
\end{aligned}
$$

The region occupied by the neutron halo is limited in extent by the requirement that virial temperature at the edge of the halo approaches helium dissociation threshold $\simeq 20 \mathrm{MeV}$. The latter means $R / R_{\mathrm{g}} \lesssim 30$. Substituting this ratio in Eq. (9) one finds $\dot{M}_{\text {min }} \sim 10^{-3} \dot{M}_{\max }$ for a black hole of ten solar masses and $\eta_{\mathrm{He}}=0.2$. Thus, there is a large parameter space in which the neutron halo may exist. It should be noted that the halo can be formed even if $\dot{M}<\dot{M}_{\text {min }}$, but with a smaller radius. In principle, this marginal solution could exist up to the accretion rates as low as $\sim 0.03 \dot{M}_{\text {min }}$, but in this case it faces a serious threat from rapidly increasing neutron losses in the immediate vicinity of the last stable orbit. This regime, as well as the dynamics of neutron halo in the limit of prevailing neutron-neutron collisions, will be considered elsewhere.

\section{Virialization of neutrons in the halo}

As was mentioned above, precession of neutron's orbit caused by the gravimagnetic field of a rotating black hole results in additional heating of neutrons and may lead to complete virialization of the neutron halo if the precession frequency is higher than or of the order of one half of the neutron-proton collision rate, i.e.,

$\frac{a c}{2 \pi R_{\mathrm{g}}}\left(\frac{R_{\mathrm{g}}}{R}\right)^{3} \gtrsim \frac{\nu_{\mathrm{pn}}}{2}$,

where $a$ is the black hole angular momentum in units $M R_{\mathrm{g}} c / 2$. Substituting $\nu_{\mathrm{pn}}$ in the above equation it is easy to find the corresponding inequality for the accretion rate:

$\dot{M} \lesssim \frac{a}{\sqrt{2} \pi}\left(\frac{R_{\mathrm{g}}}{R}\right)^{3 / 2} \dot{M}_{\max }$

Even for a rapidly rotating $(a \rightarrow 1)$ black hole Eq. (11) requires mass accretion rate hardly consistent with the lower limit $\dot{M}_{\min }$. Therefore, we conclude that the mechanism under consideration is unlikely to cause virialization of the neutron halo close to its outer edge. However, the virialization is possible in the inner part of the halo at the distance

$R \lesssim\left(\frac{a}{\sqrt{2} \pi} \frac{\dot{M}_{\max }}{\dot{M}}\right)^{2 / 3} R_{\mathrm{g}}$

\section{Long-term variability}

As the neutron halo close to the black hole is likely to be virialized, it produces a neutron wind which is nearly isotropic, expands with a velocity comparable to the velocity of light, and may carry about a half of all neutrons extracted from helium in an accretion disk. Note that helium nuclei completely dissociate when the accretion rate is capable to support a self-sustained neutron halo or is comparable with $\dot{M}_{\max }$, i.e. at any $\dot{M} \gtrsim \dot{M}_{\min }$. The neutron wind exerts a dynamical pressure on the infalling material via the protons originating from the neutron decay. At a distance smaller than $t_{\mathrm{n}} V_{\mathrm{w}}$ ( $V_{\mathrm{w}}$ is a typical velocity of neutrons in the wind) this pressure may be expressed in the following form

$P_{\mathrm{w}} \simeq \frac{\eta_{\mathrm{He}}}{4} \frac{\dot{M}}{4 \pi R t_{\mathrm{n}}}$ 
In the case of quasi-spherical accretion (e.g., from stellar wind emanating from a massive companion star) the dynamical pressure of the neutron wind prevents the surrounding matter from infall provided the dynamical pressure of an accreted plasma at the sonic point $R_{\mathrm{s}} \simeq$ $\left(c / V_{\infty}\right)^{2} R_{\mathrm{g}} \quad\left(V_{\infty}\right.$ is the velocity of the black hole with respect to the stellar wind is less than $P_{\mathrm{w}}$. This gives the condition

$\frac{\dot{M} V_{\infty}}{4 \pi R_{\mathrm{s}}^{2}} \lesssim P_{\mathrm{w}} \Rightarrow \frac{V_{\infty}}{c} \lesssim\left(\frac{\eta_{\mathrm{He}}}{4} \frac{R_{\mathrm{g}}}{c t_{\mathrm{n}}}\right)^{1 / 3}$.

As soon as the condition (14) is satisfied, the accretion stops, but the source remains active thanks to the matter accumulated in an accretion disk. The disk becomes exhausted in a time approximately equal to its viscous timescale at the outer radius, and after that the source switches off allowing for a new cycle of accretion from a stellar wind. The quiet period lasts for roughly the same time as the active one, until the matter initially deposited at the edge of the disk reaches the neutron halo boundary. Such a mechanism results in a long-term variability with a period very sensitive to the disk outer size, i.e. to the angular momentum per unit mass of the infalling material. The maximum period may be hundreds or thousands years.

\section{Proton-neutron bremsstrahlung}

Some of the proton-neutron collisions result in the emission of gamma-quanta, either in deuterium formation process or due to bremsstrahlung, first suggested to be an efficient source of radiation in proton-neutron plasma by Aharonyan \& Sunyaev (1984). Let us define $\sigma_{\gamma}$ so that the energy radiated in each collision of a neutron with a proton constitutes on average $\sigma_{\gamma} / \sigma_{\mathrm{pn}}$ fraction of their relative kinetic energy. The typical value of this ratio is of $\sim 10^{-4}$ in our case. Therefore, the energy radiated by a neutron per unit radius is

$\frac{\mathrm{d} \epsilon_{\mathrm{n}}}{\mathrm{d} R}=\frac{\sigma_{\gamma}}{\sigma_{\mathrm{pn}}}(3 T+\Delta) \frac{\mathrm{d} N_{\mathrm{c}}}{\mathrm{d} R}$

Here $\Delta \simeq 2.2 \mathrm{MeV}$ is the binding energy of deuteron, and $T$ is an average temperature of protons and neutrons. The radiative efficiency of a disk due to proton-neutron bremsstrahlung, as follows from the above equation, is equal to

$\frac{\left(\eta_{\mathrm{He}} / 2\right) \mathrm{d} \epsilon_{\mathrm{n}} / \mathrm{d} R}{\left(R_{\mathrm{g}} / 4 R^{2}\right) m_{\mathrm{p}} c^{2}}=\eta_{\mathrm{He}} \frac{\sigma_{\gamma}}{\sigma_{\mathrm{pn}}}(3 T+\Delta) \frac{16 \pi h_{\mathrm{n}}}{\langle\sigma V\rangle_{\mathrm{pn}} \dot{M}}$.

This efficiency approaches unity when

$\dot{M} \lesssim 8 \pi \eta_{\mathrm{He}} \frac{\sigma_{\gamma}}{\sigma_{\mathrm{pn}}} \frac{R_{\mathrm{g}} m_{\mathrm{p}} c^{2}}{\langle\sigma V\rangle_{\mathrm{pn}}} \frac{h_{\mathrm{n}}}{R}=\frac{2 \eta_{\mathrm{He}}}{\alpha} \frac{\sigma_{\gamma}}{\sigma_{\mathrm{pn}}}\left(\frac{R}{h_{\mathrm{d}}}\right)^{2} \dot{M}_{\max }$,

assuming $\Delta \ll 3 T$ and $3 T \simeq\left(R_{\mathrm{g}} / 2 R\right) m_{\mathrm{p}} c^{2}$. At an accretion rate exceeding the above limit, luminosity due to proton-neutron bremsstrahlung remains constant independently on the accretion rate.

\section{Pion production and hard gamma-ray luminosity of the halo}

As was mentioned above, the inner part of the neutron halo is most likely to be virialized. Therefore, neutronproton and neutron-neutron collisions within $R \sim 3 R_{\mathrm{g}}$ lead to an efficient production of pions. If we assume for simplicity that the mean pion-production rate $\xi_{\pi}=$ $\left\langle\sigma_{\pi} V\right\rangle$, averaged over the velocity distribution of neutrons, is the same for both kinds of collisions, the total luminosity in pions is

$L_{\pi} \sim \xi_{\pi}\left(\frac{1}{2} n_{\mathrm{n}}^{2}+\frac{h_{\mathrm{d}}}{h_{\mathrm{n}}} n_{\mathrm{n}} n_{\mathrm{p}}\right) R_{\pi}^{3} E_{\pi}$,

where $R_{\pi}$ is the radius within which the pions are produced, $E_{\pi} \simeq 140 \mathrm{MeV}$ is the pion energy. If He dissociation is complete, the density of neutrons in the inner halo can be found from the following balance condition:

$\frac{\eta_{\mathrm{He}} \dot{M}}{2 m_{\mathrm{p}}}=\xi_{\operatorname{loss}} \frac{n_{\mathrm{n}}^{2}}{2} R_{\pi}^{3}$,

where the rate of neutron losses $\xi_{\text {loss }}$ is mainly determined by the scattering into a black hole. Using the expression for $n_{\mathrm{n}}$ from Eq. (19), the expression for $n_{\mathrm{p}}$ derived from the mass continuity equation, and assuming $h_{\mathrm{n}}=R$, we obtain pion luminosities associated with neutron-neutron and neutron-proton collisions:

$L_{\mathrm{nn}}=\frac{\xi_{\pi}}{\xi_{\text {loss }}} \frac{\eta_{\mathrm{He}} \dot{M} E_{\pi}}{2 m_{\mathrm{p}}}$

$L_{\mathrm{pn}}=\frac{E_{\pi}}{2 \pi \alpha} \frac{\xi_{\pi}}{\xi_{\text {loss }}}\left(\frac{R}{h_{\mathrm{d}}}\right)^{2}\left(\frac{\eta_{\mathrm{He}} \xi_{\mathrm{loss}} \dot{M}^{3}}{R_{\mathrm{g}} c^{2} m_{\mathrm{p}}^{3}}\right)^{1 / 2}$.

The upper limit on luminosities can be obtained by putting $\dot{M}=\dot{M}_{\max }$. This gives

$L_{\mathrm{nn}}^{\max } \simeq 7 \times 10^{38} \alpha \eta_{\mathrm{He}} M_{10}\left(\frac{h_{\mathrm{d}}}{R}\right)^{2} \mathrm{erg} / \mathrm{s}$,

$L_{\mathrm{pn}}^{\max } \simeq 10^{38} M_{10} \sqrt{\alpha \eta_{\mathrm{He}}}\left(\frac{h_{\mathrm{d}}}{R}\right) \mathrm{erg} / \mathrm{s}$,

where $M_{10}$ is the black-hole mass in units of 10 solar masses. Approximately $1 / 2$ of the total pion luminosity goes to gamma-quanta and $\mathrm{e}^{-} \mathrm{e}^{+}$pairs and can be transformed to radiation via inverse Compton scattering of soft $\mathrm{X}$-rays and electromagnetic cascade.

In the presence of abundant soft X-rays from disk, relativistic $\mathrm{e}^{-} \mathrm{e}^{+}$pairs are quickly cooled, and the snapshot of electron distribution would demonstrate that the average energy is between $50-500 \mathrm{keV}$. The resulting photon spectrum above $100 \mathrm{keV}$ has a typical comptonization pattern. Pair cascade, if developed, leads to a hard power-law tail with photon index around 2. Both features are frequently observed in the hard state of accreting black-hole candidates. In addition, very hard radiation should be present with photon energies up to $70 \mathrm{MeV}$, but it is expected to 
contain much less energy than hard X-rays and is difficult to observe.

Neutron halo can be also an efficient source of deuterium line at $\simeq 2.2 \mathrm{MeV}$. It originates from radiative capture of neutrons that evaporate from the halo and are intercepted by the cold outer disk. The total luminosity in the line is $\beta \eta_{\mathrm{He}} \dot{M} \Delta /\left(2 m_{\mathrm{p}}\right)$, where $\beta$ is the fraction of neutrons that are captured in the outer disk. Its value depends critically on the velocity distribution of neutrons and the size of the halo. For the optimistic value $\beta \sim 1 / 2$ we have $L(2.2 \mathrm{MeV}) \sim 10^{-4} \dot{M} c^{2}$ that can be as high as $10^{34} \mathrm{erg} / \mathrm{s}$ for $\dot{M}$ close to $\dot{M}_{\max }$. This is detectable by the future INTEGRAL mission from a distance of up to several kpc.

\section{Conclusions}

We suggest and analyze in detail the possibility of selfsustained neutron halo formation in the vicinity of a diskaccreting black hole (or a neutron star). The neutrons come from collisional dissociation of $\mathrm{He}$ in the infalling matter. The term "self-sustained" means here that, even if the ion temperature in the disk is below the threshold for He dissociation by protons, the neutron production is supported by collisions of energetic neutrons from the halo with helium nuclei.

Our analysis shows that halo formation is possible when the mass accretion rate is below some critical value. In this case neutrons accrete slower than ions, which leads to the neutron pile-up: the density of neutrons can be much higher than proton density. Neutron halo exists in a broad range of disk parameters and can lead to the number of observational and dynamical effects including selfregulation of accretion and long-term variability of the sources, enhanced hard X-ray and gamma-ray luminosity (also due to proton-neutron bremsstrahlung), $2.2 \mathrm{MeV}$ deuterium gamma-ray line resulting from neutron capture.

Acknowledgements. We thank Yuri Lyubarskii for stimulating discussions and the referee, M. Medvedev, for useful comments. This work has been supported in part by the Russian Foundation for Basic Research through grants 99-02-18244 and 01-02-06511.

\section{References}

Aharonyan, F. A., \& Sunyaev, R. A. 1984, MNRAS, 210, 257 Belyanin, A. A. 1999, A\&A, 344, 1999

Bouchet, L., Mandrou, P., Roques, J. P., et al. 1991, ApJ, 383, L45

Churazov, E., Gilfanov, M., Sunyaev, R., et al. 1993, ApJ, 407, 752

Guessoum, N., \& Kazanas, D. 1989, ApJ, 345, 356

Li, H., \& Liang, E. P. 1996, ApJ, 458, 514

Mirabel, I. F., \& Rodríguez, L. F. 1998, Nature, 392, 673

Narayan, R., McClintock, J., \& Yi, I. 1996, ApJ, 457, 821

Narayan, R., Barret, D., \& McClintock, J. 1997, ApJ, 482, 448

Shapiro, S. L., Lightman, A. P., \& Eardley, D. M. 1976, ApJ, 204, 187 